\title{
Surgical management of duodenal stump fistula after elective gastrectomy for malignancy: an Italian retrospective multicenter study
}

\author{
Luca Cozzaglio • Marco Giovenzana $\cdot$ Roberto Biffi $\cdot$ Lorenzo Cobianchi $\cdot$ Arianna Coniglio • \\ Massimo Framarini - Leonardo Gerard • Luca Gianotti • Alberto Marchet • Vincenzo Mazzaferro • \\ Paolo Morgagni - Elena Orsenigo - Stefano Rausei - Fabrizio Romano - Fausto Rosa - Riccardo Rosati • \\ Francesco Roviello • Matteo Sacchi • Emanuela Morenghi • Vittorio Quagliuolo
}

Received: 20 May 2014/ Accepted: 17 November 2014/Published online: 10 December 2014

(C) The International Gastric Cancer Association and The Japanese Gastric Cancer Association 2014

\begin{abstract}
Background Duodenal stump fistula (DSF) is a severe complication of gastrectomy. Although nonsurgical therapy is preferred, surgery is still mandatory in one third of DSF patients. The aim of this article is to analyze the surgical management of DSF and factors related to its outcome.

Methods We performed a retrospective multicenter study using data from January 1990 to November 2011 in 16
\end{abstract}

On behalf of the Italian Research Group for Gastric Cancer, GIRCG.

L. Cozzaglio $(\bowtie) \cdot$ M. Giovenzana $\cdot$ V. Quagliuolo

Division of Surgical Oncology, Humanitas Clinical and

Research Center, Via Manzoni 56, 20089 Rozzano, MI, Italy

e-mail: felugia@libero.it; luca.cozzaglio@humanitas.it

R. Biffi

Division of Abdominal-Pelvic and Minimally Invasive Surgery,

European Institute of Oncology, Milan, Italy

L. Cobianchi

Division of General Surgery 1, IRCCS Fondazione Policlinico S.

Matteo, University of Pavia, Pavia, Italy

A. Coniglio

Department of Clinical and Experimental Sciences, University of Brescia, Brescia, Italy

M. Framarini

Division of Surgery and Advanced Oncological Therapies, G.B.

Morgagni-L. Pierantoni Hospital, Forlì, Italy

L. Gerard

Division of Surgery, C. Poma Hospital, Mantua, Italy

L. Gianotti · F. Romano

Unit of Hepatobiliopancreatic Surgery, Department of Surgery and Translational Medicine, S. Gerardo Hospital, University of Milan-Bicocca, Monza, Italy
Italian surgery centers. We collected 8,268 elective gastrectomies for malignancies, 7,987 by the laparotomic and 281 by the laparoscopic approach. Two hundred five patients developed a DSF, 75 of whom underwent surgery for DSF. We analyzed mortality and DSF healing time as well as the impact of clinical, oncological, and surgical characteristics.

Results The laparoscopic approach increased the risk of DSF development (odds ratio 5.6, $95 \%$ confidence interval $2.7-10.6, P<0.001)$. The indication for first DSF surgery was intra-abdominal sepsis; the failure rate was over $30 \%$,

A. Marchet

Department of Surgical Science, University of Padua, Padua, Italy

V. Mazzaferro

Division of Gastrointestinal Surgery and Liver Transplantation, IRCCS Istituto Nazionale per lo Studio e la Cura dei Tumori, Milan, Italy

P. Morgagni

Division of Surgery, G.B. Morgagni-L.Pierantoni Hospital, Forlì, Italy

\section{E. Orsenigo}

Department of Surgery, Vita-Salute San Raffaele University, Milan, Italy

\section{S. Rausei}

Department of Surgical Science, Insubria University, Varese, Italy

\section{F. Rosa}

Division of Digestive Surgery, Department of Surgical Sciences, Policlinico A. Gemelli, Catholic University Sacro Cuore, Rome, Italy 
associated with the appearance of fistulas of neighboring organs, bleeding, and the need for reoperations. The mortality rate was $28 \%$ and was related to the presence of vascular disease $(P=0.04)$, more than one reoperation $(P=0.05)$, sepsis $(P<0.001)$, and renal failure $(P<0.001)$. Fifty-four patients recovered after a median of 39 days (interquartile range 22-68 days); the need to perform more reoperations $(P<0.01)$ and the presence of an abdominal abscess $(P<0.01)$ led to an increase in healing time.

Conclusions Surgery for DSF has a poor prognosis. Our data will help to identify patients at risk of death, but unfortunately could not establish the best surgical procedure applicable to all cases of DSF.

Keywords Gastrectomy · Complications · Duodenal stump fistula $\cdot$ Surgery

\section{Introduction}

Duodenal stump fistula (DSF) after gastrectomy is a severe complication with high morbidity and mortality and a very long period of hospitalization; DSF frequency ranges from 1.6 to $5 \%$ and the mortality rate ranges from 16 to $20 \%$ [1-4].

DSF is often difficult to treat because of the highly enzyme-rich duodenal juice and deep location of the fistula. In a previous retrospective multicenter study, we analyzed 3,685 patients undergoing gastrectomy for malignancies who developed 68 DSFs [4], and showed that DSF features had changed in the last 30 years. DSF alone no longer leads to death; in fact, some complications observed in the past, such as fluid and electrolyte loss and dermatitis, have disappeared owing to improvements, in particular parenteral nutrition and wound care. However, additional new complications such as bleeding and fistulas of neighboring organs were emerging [4]. Although medical therapy is associated with better outcomes [4], surgery

\section{R. Rosati}

Division of General and Minimally Invasive Surgery, Humanitas Clinical and Research Center, Rozzano, MI, Italy

F. Roviello

Division of Surgical Oncology, Department of Human Pathology and Oncology, University of Siena, Siena, Italy

M. Sacchi

Division of General Surgery, Humanitas Clinical and Research Center, University of Milan, Rozzano, MI, Italy

E. Morenghi

Department of Biostatistics, Humanitas Clinical and Research Center, Rozzano, MI, Italy is still mandatory in cases of severe abdominal sepsis or bleeding that are not otherwise manageable. However, reoperation is often ineffective owing to postoperative edema and inflammation, and the prognosis of patients undergoing surgery for DSF remains very poor [5]. To improve the outcome of these patients, many surgical procedures have been proposed-from washing the peritoneal cavity and abdominal drainage to tube duodenostomy [6], closure of the fistula, fistula repair with a rectus abdominis flap [7], fistula closure by Roux-en-Y duodenojejunostomy [8], biliogastric diversion [9], laparostomy, and pancreatoduodenectomy [10]—but surgeons are often unsure about the best management. The main aim of this study was to analyze the surgical indications for DSF, the type of surgery, possible complications, and outcome. Secondary aims were to investigate the presence of prognostic factors related to outcome in DSF patients undergoing surgery, and to define the best surgical management of DSF. Considering the rarity of this complication, we performed a multicenter study allowing extensive accrual.

\section{Methods}

We performed a multicenter retrospective study involving several Italian surgical units with particular experience in gastric surgery. The inclusion criterion was as follows: patients with gastric malignancies undergoing elective gastrectomy with duodenal stump who developed DSF and were then resubmitted to surgery. The diagnosis of DSF was based on the presence of duodenal juice in the surgical drainage or its leakage through the surgical abdominal incision, and was confirmed by CT scan and/or fistulography, or by surgical exploration in the operating room. The indication and timing of surgery for DSF were determined by each surgical team. No exclusion criteria were applied. We collected general data from each center on all patients undergoing gastrectomy for malignancies during the study period and all occurring DSFs, and we analyzed those patients who underwent surgery for DSF. For the latter group, we collected a series of data regarding clinical characteristics (age, gender, comorbidities, serum albumin level, serum lymphocyte count, 10 and $20 \%$ weight loss), oncological characteristics (histological features, preoperative chemotherapy, neoplastic duodenal stump infiltration, stage), gastrectomy characteristics (laparotomic or laparoscopic access, subtotal or total gastrectomy, digestive tract reconstruction, manual oversewing of the duodenal stump, extent of lymphadenectomy), DSF characteristics (time of onset, output, healing time), DSF treatment (number of reoperations, type of reoperation, postoperative timing of reoperation, oral nutrition, enteral nutrition, parenteral nutrition, 
octreotide/somatostatin administration, percutaneous procedures), and details on the postoperative course (number and type of other complications, outcome).

Resolution of DSF had to be proved by clinical judgment and the absence of any drainage or residual percutaneous fistula, and was confirmed by CT scan and/or fistulography. The healing time was calculated on the basis of the criteria for DSF resolution reported above, including relapses and repeated hospital admissions.

\section{Statistical analysis}

Patient data were described as number and percentage, mean and standard deviation, or median and interquartile range (IQR), as appropriate. To assess the association between DSF onset and the type of gastrectomy (laparotomic vs laparoscopic), we used odds ratios (OR) and $95 \%$ confidence intervals (CI). To assess the associations between mortality and patient characteristics (clinical, oncological, and surgical), characteristics of DSF, therapies, and the presence of other complications, we used Pearson's $\chi^{2}$ test (with Fisher's correction when necessary), the two-tailed $t$ test, or Wilcoxon's rank-sum test, as appropriate. To assess the associations between healing time and clinical and oncological characteristics of the patients, characteristics of DSF, therapies, and the presence of other complications, we used Wilcoxon's rank-sum test; in this case we excluded one outlier for healing time. To explore the associations between mortality and covariates, we used the Cox regression model; all results were adjusted for age and the number of comorbidities. The overall survival time was calculated from the date of DSF onset to the date of death. $P$ values below 0.05 were considered statistically significant. All analyses were performed using Stata 11 (StataCorp, College Station, TX, USA).

\section{Results}

We collected 8,268 elective gastrectomies for malignancies from January 1990 to November 2011 in 16 Italian centers, 7,987 by the laparotomic and 281 by the laparoscopic approach. Two hundred five patients developed a postoperative DSF, 75 of whom underwent surgery for DSF (median cumulative incidence in the different centers $2.29 \%$, IQR 1.13-4.86 \%). The laparoscopic approach was used in five centers, with a median of 17 laparoscopic gastrectomies per center (IQR 16-41). The cumulative incidence of DSFs requiring reoperation was higher in the laparoscopy group than the laparotomy group, 4.3 and $0.8 \%$, respectively $(P<0.001)$, with an OR of $5.6(95 \%$ CI $2.7-10.6, P<0.001)$. The target of this study was the group of 75 patients who underwent surgery for DSF.
Histological features included 72 carcinomas, two gastrointestinal stromal tumors, and one lymphoma; 46 patients had regional lymph node metastases, 11 had metastastic disease, and three had received preoperative chemotherapy. The characteristics of the patients are reported in Table 1. For digestive tract reconstruction after total gastrectomy, all centers employed Roux-en-Y reconstruction, whereas Billroth II reconstruction (13 patients, $25.5 \%$ ) or Roux-enY reconstruction (38 patients, $74.5 \%$ ) was used for subtotal gastrectomy. The duodenal stump was closed by a stapler: GIA in 40 patients, TA in 22 patients, Endo GIA in 12 patients, and an unspecified device in one patient. Manual oversewing was added in 50 patients $(66.7 \%)$, depending mostly on the habit of the surgeon rather than on stapler-related problems; in fact, in 11 of 16 centers, manual oversewing was performed routinely. Furthermore, the surgical approach did not influence the surgeons' habits, and the rate of manual oversewing in laparoscopic

Table 1 Characteristics of the series

\begin{tabular}{ll}
\hline Number of patients & 75 \\
Male/female & $52 / 23$ \\
Mean age \pm SD (years) & $69.1 \pm 8.2$ \\
Weight loss $>10 \%$ & $24(32.0 \%)$ \\
Weight loss $>20 \%$ & $4(5.3 \%)$ \\
Serum albumin level (g/L) & 37 (median); 33-42 (IQR) \\
Serum lymphocyte count $\left(\mathrm{n} / \mathrm{mm}^{3}\right)$ & $1,870($ median); 1,450-2,300 \\
& $(\mathrm{IQR})$ \\
Comorbidities & \\
0 & $16(21.4 \%)$ \\
1 & $25(33.3 \%)$ \\
2 & $18(24.0 \%)$ \\
3 & $9(12.0 \%)$ \\
4 & $7(9.3 \%)$ \\
Neoplastic duodenal stump & $7(9.3 \%)$ \\
infiltration & \\
Laparotomic/laparoscopic access & $63 / 12$ \\
Total/subtotal gastrectomy & $24(32.0 \%) / 51(68.0 \%)$ \\
Lymphadenectomy & \\
D1 & $28(37.3 \%)$ \\
D2 & $44(58.7 \%)$ \\
D3 & $2(2.7 \%)$ \\
Unknown & $1(1.3 \%)$ \\
\hline
\end{tabular}

The comorbidities considered were arterial hypertension, diabetes mellitus, vasculopathies, heart diseases, chronic bronchitis, chronic hepatitis/cirrhosis, chronic renal failure (serum creatinine level increase above normal), and a miscellaneous group of other diseases including Raynaud's disease, Ménière's syndrome, Alzheimer's disease, hyperthyroidism, hyperuricemia, cholelithiasis, and prostate hypertrophy

$I Q R$ interquartile range, $S D$ standard deviation

a the numbers refer to the total number of comorbidities affecting each patient before gastrectomy 
and laparotomic cases was similar: 58 and $66 \%$, respectively. Three-layer staplers, absorbable polymer membranes, and nonabsorbable pericardial strips were never used.The median time of DSF onset was on postoperative day 6 (IQR 2-11 days), and the median daily output was $300 \mathrm{~mL}$ (IQR $100-750 \mathrm{~mL}$ ). The first reoperation was performed after a median of 9 days (IQR 4-16 days) from gastrectomy and a median of 1 day (IQR $0-3$ days) from DSF onset. The indication for reoperation was the development of abdominal sepsis in all patients but one, in whom the indication was failure of the DSF to heal. However, 25 patients $(33.3 \%)$ underwent a second reoperation after a median of 10 days (IQR 5-17 days) from DSF onset, 12 patients underwent a third reoperation after a median of 18 days (IQR 12.5-41.5 days) from DSF onset, four patients underwent a fourth reoperation after a median of 20 days (IQR 10-72.5 days) from DSF onset, and one patient underwent six operations.

Peritoneal washing and abdominal drainage were performed in all reoperations, and were often associated with other procedures. Table 2 shows details of the surgical procedures performed during the first, second, and third reoperations. In eight patients fibrin glue or fibrinogen and a thrombin patch were added to the duodenal suture. The four patients who had a fourth reoperation underwent necrosectomy and closure of the laparostomy; abdominal drainage and duodenal suture; abdominal drainage and vacuum therapy; hemostasis and colectomy, respectively.

Table 2 Type of surgery performed during the first, second, and third reoperations

\begin{tabular}{lcll}
\hline Surgery & $\begin{array}{l}\text { 1st } \\
\text { reoperation }\end{array}$ & $\begin{array}{l}\text { 2nd } \\
\text { reoperation }\end{array}$ & $\begin{array}{l}\text { 3rd } \\
\text { reoperation }\end{array}$ \\
\hline Number of patients & 75 & 25 & 12 \\
Drainage & $75(100 \%)$ & $23(92.0 \%)$ & $10(83.0 \%)$ \\
Duodenal suture & $38(50.7 \%)$ & $5(20.0 \%)$ & $2(16.7 \%)$ \\
Duodenostomy & $15(20.0 \%)$ & $3(12.0 \%)$ & $1(8.3 \%)$ \\
Nutritional jejunostomy & $13(17.3 \%)$ & $2(8.0 \%)$ & 0 \\
Biliary tree procedures & $14(18.7 \%)$ & $1(4.0 \%)$ & $1(8.3 \%)$ \\
Hemostasis & $3(4.0 \%)$ & $5(20.0 \%)$ & 0 \\
Laparostomy & $3(4.0 \%)$ & $1(4.0 \%)$ & $1(8.3 \%)$ \\
Operations for other & 0 & $6(24.0 \%)$ & $4(33.3 \%)$ \\
digestive fistulas & & & \\
Other procedures & $10(13.3 \%)$ & $3(12.0 \%)$ & $2(16.7 \%)$ \\
\hline
\end{tabular}

Biliary tree procedures included cholecystectomy, intracystic or intracholedochal Kehr T-tube placement, and cholecystojejunal anastomosis. Operations for other digestive fistulas included near-total gastrectomy, conversion from Billroth II to Roux-en-Y digestive reconstruction, gastrojejunal anastomosis or gastrojejunal bypass, gastric derotation, jejunal anastomosis, ileostomy, colectomy, and colostomy. Other procedures included cutting peritoneal adhesions, omentopexy, splenectomy, abdominal hernia repair, ileostomy closure, peritoneal washing plus application of vacuum therapy, and tracheotomy.
With regard to nonsurgical therapies, 35 patients $(47.7 \%)$ were given octreotide or somatostatin, 21 $(28.0 \%)$ received oral nutrition, $31(41.3 \%)$ received enteral nutrition, and $69(92.0 \%)$ received parenteral nutrition. Percutaneous abdominal abscess drainage was performed in 18 patients $(24.0 \%)$, in nine before and in nine after reoperation for DSF, and percutaneous transhepatic biliary drainage was performed in eight patients $(10 \%)$, in two before and in six after reoperation for DSF.

Complications were very common and occurred in 74 of 75 patients. Many patients developed more than one complication; the details are reported in Table 3. Development of digestive fistulas of neighboring organs secondary to persistence of DSF involved the colon (11 patients), pancreas (four patients), esophagojejunal anastomosis (three patients), gastrojejunal anastomosis (three patients), and jejunojejunal anastomosis (one patient); in two cases a colonic fistula was associated with a gastrojejunal anastomosis fistula, and in one case a jejunojejunal anastomosis fistula was associated with an esophagojejunal anastomosis fistula.

Eighteen patients developed intra-abdominal bleeding, which was associated with intra-abdominal sepsis in all but three patients. Of these three patients, one had acute pancreatitis and one had a jejunal loop rotation. All patients underwent emergency reoperation for their critical status related to bleeding (nine patients) or sepsis (nine patients). Interventional radiology for abdominal bleeding was performed in only one patient and without success.

Fifty-four patients $(72.0 \%)$ recovered after a median of 39 days (IQR 22-68 days) since DSF onset. The overall mortality rate was $28.0 \%$ (21 patients) after a median of 32 days (IQR 18-41 days) since DSF onset; death was due to multiple organ failure in 20 patients and intra-abdominal

Table 3 Complications

\begin{tabular}{lll}
\hline Type of complication & $\begin{array}{l}\text { Number of } \\
\text { patients }\end{array}$ & $\begin{array}{l}\text { Percentage of } \\
\text { patients }\end{array}$ \\
\hline Abdominal abscess & 53 & 70.7 \\
Sepsis & 46 & 61.3 \\
Pneumonia & 33 & 44.0 \\
Surgical site infection & 29 & 38.7 \\
Digestive fistulas & 22 & 29.3 \\
Acute renal failure & 21 & 28.0 \\
Intra-abdominal bleeding & 18 & 24.0 \\
Central line infection & 13 & 17.3 \\
Acute pancreatitis & 7 & 9.3 \\
Abdominal wall necrosis & 6 & 8.0 \\
Other & 16 & 21.3
\end{tabular}

Other includes septic arthritis, pleuritis, dermatitis, fasciitis, hypertensive attacks, cerebral ischemia, neuropathy, pulmonary embolism, pneumothorax, bowel occlusion, and Roux-en-Y syndrome. 
bleeding in one patient. In an attempt to detect the prognostic factors related to outcome and healing time, we analyzed all clinical and oncological characteristics of the patients, the characteristics of gastrectomy and DSF, the therapies used (Tables 1,2), and details of the postoperative course (Table 3 ).

Analysis of prognostic factors related to postoperative mortality

In the univariate analysis we found a correlation between mortality and the presence of vascular disease (mortality rate $62.5 \%$ vs $23.9 \%$ in its presence and absence, respectively; $P=0.04$ ), and between mortality and the number of reoperations (mortality rate $44.0 \%$ vs $20.0 \%$ for two or more operations and zero operations or one operation, respectively; $P=0.05$ ). Moreover, mortality was related to sepsis (mortality rate $43.5 \%$ vs $3.5 \%$ for septic and nonseptic patients, respectively; $P<0.001$ ) and acute renal failure (mortality rate $61.9 \%$ vs $14.8 \%$ for its presence and absence, respectively; $P<0.001$ ); when sepsis was associated with acute renal failure, the mortality rate reached $75 \%$. Furthermore, none of the patients who recovered developed acute renal failure. We also found some clinically interesting differences that almost reached statistical significance: age $(72.0 \pm 7.9$ years vs $68.0 \pm 8.2$ years in patients who died and recovered, respectively; $P=0.06$ ) and number of comorbidities (mortality rate $50.0 \%$ vs $22.0 \%$ for three to four comorbidities and zero to two comorbidities, respectively; $P=0.056$ ).

In the Cox regression analysis we found similar results (Table 4) after adjusting for age and a number of comorbidities $>3$, finding a hazard ratio (HR) significantly different from 1 for sepsis (HR 13.8, $95 \%$ CI 1.8-103.9, $P=0.01$ ), acute renal failure (HR 6.3, $95 \%$ CI 2.3-17.5, $P<0.001$ ), and four reoperations with respect to one reoperation (HR 6.4, $95 \%$ CI 1.6-26.5, $P=0.01$ ).

Lastly, we found a cluster of characteristics showing a clinically interesting difference without reaching statistical significance: presence of lymph node metastasis (mortality rate $36.4 \%$ vs $26.2 \%$ for its presence and absence, respectively); acute pancreatitis ( $57.1 \%$ vs $25.0 \%$ for its presence and absence, respectively); abdominal wall necrosis $(50.0 \%$ vs $26.0 \%$ for its presence and absence, respectively); intra-abdominal bleeding ( $38.9 \%$ vs $24.6 \%$ for its presence and absence, respectively), and pneumonia (36.3\% vs $21.4 \%$ for its presence and absence, respectively). With regard to DSF treatment, we found that patients had a better prognosis, without reaching statistical significance, when peritoneal washing and abdominal drainage were combined with a surgical or percutaneous procedure on the biliary tree to carry the bile outside
Table 4 Cox regression analysis

\begin{tabular}{lcc}
\hline & Hazard ratio ${ }^{\text {a }}$ & $P$ \\
\hline Sex (male) & $1.4(0.5-3.9)$ & 0.48 \\
Gastrectomy (total) & $1.0(0.1-4.2)$ & 0.45 \\
Weight loss >10 \% (yes) & $1.3(0.3-3.4)$ & 0.47 \\
T stage (3-4) & $2.6(0.9-7.3)$ & 0.08 \\
N stage (1) & $1.6(0.6-4.4)$ & 0.32 \\
Biliary procedures (yes) & $0.6(0.2-1.7)$ & 0.35 \\
Number of complications & $1.2(0.9-1.4)$ & 0.18 \\
Abdominal abscess (yes) & $0.5(0.2-1.4)$ & 0.17 \\
Sepsis (yes) & $13.8(1.8-103.9)$ & 0.01 \\
Pneumonia (yes) & $1.5(0.6-3.9)$ & 0.37 \\
Surgical site infection (yes) & $1.5(0.6-3.8)$ & 0.36 \\
Acute renal failure (yes) & $6.3(2.3-17.5)$ & $<0.001$ \\
Fistulas (yes) & $0.4(0.1-1.3)$ & 0.12 \\
Intra-abdominal bleeding (yes) & $1.5(0.6-3.7)$ & 0.42 \\
Central line infection (yes) & $0.4(0.1-1.8)$ & 0.23 \\
Acute pancreatitis (yes) & $2.9(0.9-9.3)$ & 0.06 \\
Number of reoperations & & \\
1 & 1 & 0.45 \\
2 & $1.6(0.5-4.9)$ & 0.50 \\
3 & $1.6(0.4-6.4)$ & \\
4 & $6.4(1.6-26.5)$ & 0.01 \\
\hline
\end{tabular}

${ }^{a}$ The $95 \%$ confidence interval is given in parentheses.

(mortality rate $31.2 \%$ vs $14.3 \%$ without and with a procedure on the biliary tree, respectively). The need for parenteral nutrition seemed to increase the mortality rate: 21 of 69 patients $(30.4 \%)$ who received parenteral nutrition died, whereas all six patients who did not receive parenteral nutrition recovered.

Analysis of healing time

Although 54 patients recovered, the analysis was performed on 53 patients because we excluded one outlier for healing time. The healing time increased with the number of reoperations: the median time was 28.5 days (IQR 18-60 days) in 40 patients with one reoperation versus 63 days (IQR 50-82 days) in 13 patients with two or more reoperations $(P=0.01)$. The presence of an abdominal abscess led to an increase in healing time from 25 days (IQR 15-37 days) in 17 patients with no abscess to 54 days (IQR 24.5-83 days) in 36 patients with an abscess $(P=0.01)$.

Other possible causes of delay in healing were the onset of a colonic fistula [median time 36.5 days (IQR 20-61.5 days) and 60 days (IQR 47-103 days) in its absence and presence, respectively] and central line infection [median time 36 days (IQR 21-60 days) and 70 days (IQR 38-103 days) in its absence and presence, 
respectively], where we detected a trend without full statistical significance. Regarding the type of the first reoperation, the addition of another procedure to peritoneal washing and abdominal drainage reduced the healing time from 58 days (IQR 38-180 days) to 36.5 days (IQR 21-63 days), but also these data were not statistically significant.

\section{Discussion}

DSF is one of the most serious complications of gastrectomy, and is characterized by wide variation in the time of onset, output, clinical severity, risk of relapse, and outcome $[4,5]$. Mild fever associated with pain in the upper right abdomen, even many days after gastrectomy, could be indicative of DSF. Nonsurgical approaches are usually preferred $[4,5]$, in particular percutaneous procedures such as abdominal abscess drainage, transhepatic biliary drainage with [11] or without [12] an occlusion balloon, duodenostomy [13], and fistula obliteration by glue injection $[14,15]$, or endoscopic procedures such as DSF closure by clips [16] or endoloops and glue [17]. Nevertheless, in over one third of patients one or more reoperations are necessary. Babu and Finch [5] in a recent review collected 84 cases of surgical treatment of postoperative DSF, but including not only postgastrectomy patients scattered over 13 different articles with a median number of two patients per series. The large number of published case reports and the variety of proposed surgical procedures demonstrate the often improvised strategy of surgical treatment. Reoperation is usually performed early, often in an emergency setting because of acute sepsis; the type of surgery is not standardized and often depends on concomitant complications such as other digestive fistulas or intra-abdominal bleeding. Anyhow, peritoneal washing and abdominal drainage are the cornerstones of surgical DSF treatment [18]. Duodenal suturing is often useless, and in an experimental setting the addition of fibrin glue did not improve outcome [19]. Tube duodenostomy has been used for the management of DSF, but the largest series reported in the literature focused on treatment of duodenal ulcer or injuries. There are two main techniques of tube duodenostomy: end duodenostomy with the tube placed through the fistula lumen [20], and retrograde duodenostomy, where the tube is passed in a retrograde fashion through a proximal jejunal loop [21]. Some authors add a T-tube choledochostomy [20]. Needle jejunostomy for enteral nutrition and laparostomy are complementary procedures in the management of metabolic requirements and severe intra-abdominal sepsis.

The aims of this study were to analyze the indications for surgery of DSF, the type of surgery, complications, outcome, and survival, and to investigate the presence of prognostic factors related to mortality and healing time. To the best of our knowledge, this article represents the first multicenter study analyzing surgical treatment of DSF, and describes the largest series of DSFs ever published.

The data we collected on 8,268 elective gastrectomies for malignancies demonstrated that the laparoscopic approach carries a risk of DSF development requiring surgery, and although these data were related to a relatively small number (281) of laparoscopic gastrectomies with respect to laparotomic gastrectomies $(7,987)$, we calculated that the laparoscopic technique increases the risk of DSF about five times (OR 5.6; $95 \%$ CI 2.7-10.6, $P<0.001$ ). The increased risk of DSF development with the laparoscopic technique might be related to a specific learning curve, as was also suggested by other authors [22, 23]. Another hypothesis is the lack of oversewing of the duodenal stump [22], which could not be performed routinely with the laparoscopic approach, but our data do not confirm this: in fact, the rate of manual oversewing was similar in laparoscopic and laparotomic procedures. However, we have no further data on the whole population of 8,268 gastrectomies because this was not the aim of our study; therefore, this result must be confirmed in another analysis focused on the possible causes of DSF.

The indication for the first reoperation was intraabdominal sepsis; further reoperations were prompted by intra-abdominal complications related to persistence of DSF such as fistula of a neighboring organ or anastomosis, and intra-abdominal bleeding. The first reoperation was performed early, usually 1 day after DSF onset. Concerning the type of surgery, we observed a trend toward changing typology linked to the need for further reoperations (Table 2): the frequency of procedures for direct control of DSF decreased, e.g., abdominal drainage (from 100 to $83 \%$ ), duodenal suture (from 51 to $17 \%$ ), duodenostomy (from 20 to $8 \%$ ), and ancillary procedures to reduce fistula output or improve nutritional status such as procedures on the biliary tree (from 19 to $8 \%$ ) and needle jejunostomy (from 17 to $0 \%$ ), whereas the frequency of operations for other digestive fistulas on neighboring organs increased (from 4 to $33 \%$ ), as did the frequency of laparostomies (from 4 to $8 \%$ ) and other operations (from 13 to $17 \%$ ). Unfortunately, we failed to identify the best surgical strategy, probably because of the high number of surgical procedures performed and the low number of events, but our data suggest that outcome could improve if peritoneal washing and abdominal drainage were associated with a surgical or percutaneous procedure on the biliary tree.

The development of new complications was very common (Table 3), and relapses of sepsis led to an increased risk of reiterative surgery. We observed a failure rate of 
$33 \%$ after the first reoperation and a failure rate of about $50 \%$ after the second reoperation (Table 2), and found mortality to be correlated with the number of reoperations. The mortality rate of DSF patients undergoing surgery was $28 \%$; death was related to sepsis and mostly occurred in the first months after DSF onset. Sepsis and acute renal failure were often fatal, and together accounted for a mortality rate of $75 \%$. We found that vascular disease, the onset of new complications, and the need for parenteral nutrition were associated with a higher risk of death. With regard to the need for parenteral nutrition, the higher mortality rate could be linked both to the selection of patients having poor compliance with enteral or oral nutrition due to abdominal sepsis, and to central line infection. Central line infection occurred in 13 of 69 patients receiving parenteral nutrition (19\%), but its presence alone was not related to mortality, whereas in our previous article we demonstrated that the possibility of taking an oral nutrition was linked to a better outcome [4]. The data related to the healing time were similar to those reported for survival: repeated reoperations as well as septic complications such as abdominal abscess, and probably colonic fistula and central line infection, increased the healing time.

In conclusion, our study shows that when a patient requires reoperations, the outcome is negatively affected. The appearance of new complications, in particular sepsis and acute renal failure, and the need for more reoperations were linked to a poor prognosis. Our findings identified patients with a poor outcome, in particular those with vascular disease and those developing sepsis. The clinical picture of this complication is rather complex, and it was not possible to establish the most appropriate surgical procedure, applicable to all cases of DSF. The results of our study merely suggest performing surgical or percutaneous biliary drainage in association with peritoneal washing and abdominal drainage.

Acknowledgments We thank Ferdinando Cananzi for critical reading of the manuscript and giving useful advice.

\section{References}

1. Rossi JA, Sollenberger LL, Rege RV, Glenn J, Joehl RJ. External duodenal fistula. Causes, complications, and treatment. Arch Surg. 1986;12:908-12.

2. Tarazi R, Coutsoftides T, Steiger E, Fazio VW. Gastric and duodenal cutaneous fistulas. World J Surg. 1983;7:463-73.

3. Edmunds LH Jr, Williams GM, Welch CE. External fistulas arising from the gastro-intestinal tract. Ann Surg. 1960;152:445-71.

4. Cozzaglio L, Coladonato M, Biffi R, Coniglio A, Corso V, Dionigi $\mathrm{P}$, et al. Duodenal fistula after elective gastrectomy for malignant disease. An Italian retrospective multicentric study. J Gastrointest Surg. 2010;14:805-11.
5. Babu BI, Finch JG. Current status in the multidisciplinary management of duodenal fistula. Surgeon. 2013;11:158-64.

6. Levy E, Cugnenc PH, Frileux P, Hannoun N, Parc R, Huguet C, et al. Postoperative peritonitis due to gastric and duodenal fistulas. Operative management by continuous intraluminal infusion and aspiration: report of 23 cases. Br J Surg. 1984;71:543-6.

7. Chander J, Lal P, Ramteke VK. Rectus abdominis muscle flap for high-output duodenal fistula: novel technique. World J Surg. 2004;28:179-82.

8. Ujiki GT, Shields TW. Roux-en-Y operation in the management of postoperative fistula. Arch Surg. 1981;116:614-7.

9. Milias K, Deligiannidis N, Papavramidis TS, Ioannidis K, Xiros $\mathrm{N}$, Papavramidis S. Biliogastric diversion for the management of high-output duodenal fistula: report of two cases and literature review. J Gastrointest Surg. 2009;13:299-303.

10. Musicant ME, Thompson JC. The emergency management of lateral duodenal fistula by pancreaticoduodenectomy. Surg Gynecol Obstet. 1969;128:108-14.

11. Cozzaglio L, Cimino M, Mauri G, Ardito A, Pedicini V, Poretti $\mathrm{D}$, et al. Percutaneous transhepatic biliary drainage and occlusion balloon in the management of duodenal stump fistula. J Gastrointest Surg. 2011;15:1977-81.

12. Villar R, Fernàndez R, Gonzàlez J, Oliver JM, Parga G, GarcìaHidalgo E. High-output external duodenal fistula: treatment with percutaneous transhepatic biliary/duodenal drainage. Cardiovasc Intervent Radiol. 1996;19:371-3.

13. Oh JS, Lee HG, Chun HJ, Choi BG, Lee SH, Seong HT, et al. Percutaneous management of postoperative duodenal stump leakage with Foley catheter. Cardiovasc Intervent Radiol. 2013;36:1344-9.

14. Khairy GE, al-Saigh A, Trincano NS, al-Smayer S, al-Damegh S. Percutaneous obliteration of duodenal fistula. J R Coll Surg Edinb. 2000;45:342-4.

15. Bianchi A, Solduga C, Ubach M. Percutaneous obliteration of a chronic duodenal fistula. Br J Surg. 1988;75:572.

16. Bini R, Coppola F, Recchia S, Fusca M, Gaia S, Leli R. Endoscopic treatment of postgastrectomy duodenal fistula with an over-the-scope clip. Surg Innov. 2011;18:102-4.

17. Curcio G, Badas R, Miraglia R, Barresi L, Tarantino I, Traina M. Duodenal stump fistula following Roux-en-Y gastrectomy, treated with single-balloon enteroscopy using the tulip bundle technique and fibrin glue injection. Endoscopy. 2012;44(Suppl 2):E364-5.

18. Cozzaglio L, Farinella E, Coladonato M, Sciannameo F, Gennari L, Doci R. Current role of surgery in the treatment of digestive fistulas. Ann Ital Chir. 2010;81:285-94.

19. Gwozdziewicz Ł, Khan MA, Adamczyk Ł, Hać S, Rzepko R. Fibrin glue with gentamicin as an alternative to conventional surgery in experimental treatment of duodenal fistula in rats. Surg Innov. 2012;19:275-80.

20. Isik B, Yilmaz S, Kirimlioglu V, Sogutlu G, Yilmaz M, Katz D. A life-saving but inadequately discussed procedure: tube duodenostomy. Known and unknown aspects. World J Surg. 2007;31:1616-26.

21. Stone HH, Garoni WJ. Experiences in the management of duodenal wounds. South Med J. 1966;59:864-7.

22. Orsenigo E, Bissolati M, Socci C, Chiari D, Muffatti F, Nifosi J, et al. Duodenal stump fistula after gastric surgery for malignancies: a retrospective analysis of risk factors in a single centre experience. Gastric Cancer. 2014;17:733-44.

23. Kang SY, Lee SY, Kim CY, Yang DH. Comparison of learning curves and clinical outcomes between laparoscopy-assisted distal gastrectomy and open distal gastrectomy. J Gastric Cancer. 2010;49:247-53. 